\title{
Insomnia and Depressive Symptoms in Adolescents: Can Cognitive Behavioral Therapy for Insomnia Contribute to Improve Adolescents' Depressive Symptoms?
}

\author{
Weidong Song'1 Xiaohui Hu', Beifang Fan'1, Caihong Gao', Mengshi Qiu', and Jihui Zhang² \\ 'Department of Psychiatry, Shenzhen Nanshan Center for Chronic Disease Control, Nanshan, Shenzhen, China \\ 2Department of Psychiatry, Faculty of Medicine, The Chinese University of Hong Kong, Shatin, Hong Kong SAR, China
}

\begin{abstract}
Psychological and pharmacological treatments for insomnia in adolescents have been associated with reduced insomnia symptoms and improved psychological wellbeing. Many studies have suggested or illustrated that adolescents and families strongly favor cognitive behavioral therapy (CBT) and other psychotherapies over pharmacotherapy. This narrative review aimed to examine whether CBT for insomnia (CBT-I) can improve depressive symptoms in adolescents who experience both insomnia and depressive symptoms. Four studies have been found to meet the inclusion criteria in this review. There is evidence to show that CBT-I can reduce depressive symptoms in adolescents with insomnia. This finding suggest that CBT-I is not only beneficial to sleep quality but also improve mental health. However, there is still unknown whether CBT-I may treat depression in adolescents with both disorders or whether CBT-I may prevent depression in those adolescents with insomnia who are at-risk of depression.
\end{abstract}

Key Words: Insomnia; Depressive symptoms; Cognitive behavioral therapy for insomnia; Adolescents

Received: February 4, 2019 Accepted: March 25, 2019

Corresponding author: Jihui Zhang, MD, PhD, Department of Psychiatry, Faculty of Medicine, The Chinese University of Hong Kong, Shatin, Hong Kong SAR, China. Tel: 852-39197647, Fax: 852-26463325, E-mail: jihui.zhang@cuhk.edu.hk

@ This is an Open Access article distributed under the terms of the Creative Commons Attribution Non-Commercial License (https://creativecommons.org/licenses/by$\mathrm{nc} / 4.0)$ which permits unrestricted non-commercial use, distribution, and reproduction in any medium, provided the original work is properly cited.

\section{INTRODUCTION}

Epidemiology of insomnia in adolescents

Insomnia symptoms are prevalent in both adults and children [1-4]. Female sex [4], low socioeconomic status [2], stressful life events [2], and poor general and mental health [5] are commonly identified risk factors. In particular, female sex is a consistent risk factor for insomnia in adult and elderly populations in terms of the higher prevalence, more persistent natural course [6], and more serious health-related repercussions [7]. For example, stronger impacts of insomnia symptoms on pain perception [8], increased cortisol awakening response [9], and resistant hypertension [10] were found in adult females when compared with adult males.

Interestingly, most studies did not find any sex differences in insomnia in children $[1,5,6]$. Thus, puberty has been postulated as a critical stage for the development of insomnia and the emergence of the sex differences in insomnia [7-10]. Indeed, puberty is accompanied by a series of factors that may contribute to the development of insomnia, such as decreased homeostatic sleep drive and delayed circadian phase leading to a mismatch with social activity [11]. Several studies have explored the timing of sex differences in insomnia [8-10]. Johnson et al. [9] found that the onset of menses was a critical phase with an increased risk of developing insomnia in adolescent girls but pubertal maturation was not associated with increased prevalence of insomnia in adolescent boys. By using a large-scale school-based survey, we found that the prevalence of insomnia symptoms progressively increased from $3.4 \%$ to $12.2 \%$ in girls (3.6-fold) and from $4.3 \%$ to $9.1 \%$ in boys (2.1-fold) [12]. There was a significant interaction between sex and puberty in the prevalence of insomnia symptoms $(p<0.001)$ with the emergence of female preponderance at Tanner stage 4 even after controlling for age, family income, and school start time [12]. Taken together, previous studies have almost unanimously sug- 
gested that pubertal maturation plays a critical role in the onset of insomnia symptoms and the emergence of sex differences in insomnia. In this regard, this review paper will focus on insomnia in adolescents.

\section{Epidemiology of depression in adolescents and}

\section{its relationship with insomnia}

Unipolar depression is one of the most common disorders in adolescents. About $20 \%$ of adolescents have a diagnosable mental disorder, with depression being the single most common type [13]. In addition, depression increases in adolescence and occurs at double the rate in females than males, which is similar to the prevalence of insomnia in adolescents [14]. In adolescents with major depression, insomnia is the most common sleep disturbance with a rate of $72.7 \%$. Those adolescents who reported insomnia symptoms were also accompanied with more severe depression [15]. Insomnia is not just a symptom or by-product of depression, but in many cases insomnia contributes to depression onset and/or maintenance, complicates and attenuates the effectiveness of depression treatment. In addition, insomnia is the most common residual symptom in remitted depression [16].

Recently studies also suggest that insomnia is associated with an increased risk of depression in adolescents. Several studies had proven that insomnia is a risk factor for depression and can predict the development of later depression in adolescents $[17,18]$. It has been argued that insomnia in early adolescence leads to the development of later depression by altering corticolimbic circuitry possibly via the disruptive effect of hyperarousal and insufficient sleep [19], which in turn affect adolescents' emotional regulation and cognitive functioning. Others have suggested that insomnia in adolescents may reinforce ruminative thinking styles, perpetuating further insomnia symptoms, and that these processes could develop into depression over time [20]. Despite the high incidence of insomnia and the risk factor for the onset and maintenance of depression in adolescents, adolescent insomnia has received less attention in the literature [21].

On the other hand, adolescent insomnia and depression share many common features, both at the symptom level and at the disease level. There is an emerging sex difference in the prevalence of insomnia and depression in adolescents. In part, the sexual dimorphism in the prevalence of insomnia in adolescence could relate to the higher prevalence of depression in females, which also emerges after puberty [12]. Several social and biological changes occur during puberty that are sexually differentiated, such as higher stress level and greater stress reactivity in females [12] and differential changes in sex steroids associated with increased neuroendocrine sensitivity [12], which may increase risk for both depression and insomnia. Our previous study [12] has explored the associations of insomnia symptoms with lifestyle, and emotional and behavioral problems in females and males. While some relationships were common in both boys and girls, interactions with sex were also found. For example, insomnia symptoms were associated with greater consumption of energy drinks and alcohol in boys only where- as insomnia symptoms were associated with self-reported poorer emotional and behavioral wellbeing in girls only. The American Psychological Association in the 2014 nationwide "Stress in America" survey clearly indicated stress as a major concern in adolescents: perceived stress may have a detrimental effect on health by affecting mood, eating behaviors, and sleep [22].

It is common for adolescents to have and use electronic devices in their bedroom. Specifically in adolescence, the progressive delay in circadian phase results in later bedtimes leaving adolescents more time to perform various activities until late at night. One recent study from our group has also found that long time smart phone use is associated with the incidences of most sleep disturbances and mental distress, including depressive symptoms while discontinuation of long-time mobile phone use seems to alleviate these problems in youths [23]. Electronic media use at bedtime may affect both sleep and mood via the melatonin-suppression effect of bright screen light [24]. According to the comorbid and bidirectional relationship between insomnia and depression in adolescents, it is hypothesized that treating insomnia can improve depressive symptoms in adolescents with both problems. The hypothesis is based on the following reasons. First, insomnia contributes to increasing depression risk in adolescents. Adolescents who complained insomnia symptoms were more likely to report depression [odds ratio $(\mathrm{OR})=22.7][25]$ and insomnia significantly predicted future depression (hazard rate $=3.8$ ) [26]. It has been found that baseline insomnia symptoms increased the risk of anxiety and depression one year later among 2,787 adolescents in China [27]. Second, insomnia predicts the treatment outcomes of depression in adolescents. In depressed adolescents, low sleep efficiency and difficult falling sleep predicted depression recurrence following treatment [28]. Third, residual insomnia is a major component of partially remitted depression. In the Treatment of Adolescent with Depression Study (TADS), residual insomnia was the most common symptoms among depressed adolescents who had incompletely-recovered but responded to treatment [29]. This suggests that insomnia may be an important therapeutic target for treating and preventing recurrent depression in adolescents. Based on the evidences listed above, it is suggested that effective treating insomnia can improve the prognosis of depression or prevent the onset of depression in adolescents.

\section{Insomnia and depressive symptoms: shares the common characteristics}

Adolescents' depression treatments include psychological and pharmacological therapies, but the treatments are inadequacy, even in the TADS, the largest, well controlled clinical trial of adolescents' depression [30]. Many studies have proven that adolescents and their parents favor psychotherapy over pharmacotherapy [31], and cognitive behavioral therapy for insomnia (CBT-I) appears to be superior to hypnotics in the long run [32]. Metaanalysis has already supported that treating insomnia via CBT-I in both adults and elderly with depression has a positive effect on depressive symptoms $[33,34]$. To date, the literature has not been sys- 
tematically reviewed to evaluate the role of CBT-I in adolescents with comorbid insomnia and depressive symptoms. Therefore, this systematic review focuses on the following question: Can CBT-I alleviate depressive symptoms in adolescents with insomnia?

\section{TREAT THE DEPRESSION VIA CBT-I IN ADOLESCENTS}

We have conducted a comprehensive search of literature in PubMed, PsycINFO and Cochrane Controlled Register of Trial (CENTRAL). We searched for a combination of CBT-I or cognitive behavioral therapy for insomnia with depressi* and adolescen* in titles and with abstracts of using controlled vocabulary and text words in academic journal publications up to 23rd October, 2018. There are 87, 4 and 28 articles identified through searching PubMed, PsycINFO and CENTRAL respectively based on the theme that CBT-I affects or influences on depressive symptoms in adolescents who experience insomnia symptoms. Only four articles are included in our qualitative analysis and the characteristics of the included studies list in Table 1. The participants of these 4 studies were recruited from the community and/or hospital. Across studies, participants were between the ages of 11 and 20 years old, and most of the participants were female [35-38]. Two studies were randomized controlled trail $[35,38]$; the other two were pre- and post-treatments design [36,37]. The CBT-I interventions ranged from 4 to 10 sessions, and the core intervention technique consisted of an age-appropriate modification of sleep restriction, stimulus control, sleep-focused cognitive therapy and sleep hygiene education. The sample sizes included into analysis were 41, 40, 16 and 116 , respectively. All studies reported significant improvement in both insomnia symptoms and depressive symptoms [35-38]. Two studies reported effect size using Cohen's $d[36,38]$ and one using OR in depressive symptoms [35]. One study did not list the effect size and used pair wise comparisons to show that Quick Inventory of Depressive Symptoms (QIDS) scores decreased by a mean of 3.7 points from session 1 to session $5(\mathrm{SE}=1.03, \mathrm{df}=15.1, \mathrm{p}=0.027$ ) [37]. Based on the limited evidences represented above, we modestly suggest that CBT-I have a positive effect on decreasing depressive symptoms in adolescents who experience insomnia symptoms.

\section{THE MECHANISM OF CBT-I EFFECT BEYOND INSOMNIA}

The mechanism of CBT-I in treating depression may explain through circadian rhythm, Hypothalamic-pituitary-adrenal axis (HPA), and neural processing.

\section{Regularizing circadian rhythm}

Circadian rhythm is the basis of all physiological processes including mood and brain function regulation. There is growing evidences that emotions are closely linked to circadian rhythm disorders [39]. Proposed mechanism of action of CBT-I comes from the "phase-shift" model of depression. This theory posits that depres-

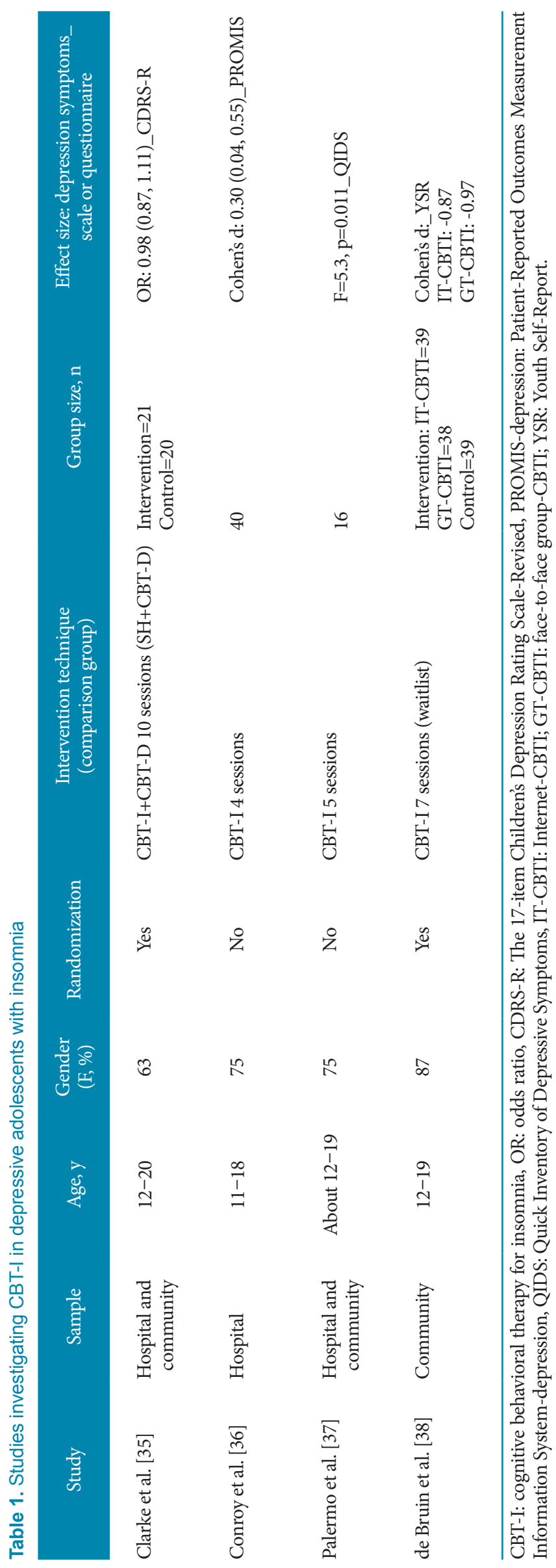


sion is at least partially a result of shifted circadian rhythms. The circadian rhythm of the central pacemaker (the suprachiasmatic nucleus) and related REM sleep, melatonin, cortisol and temperature rhythms are shifted relative to one's sleep-wake rhythm and other bodily circadian rhythms. In this case, CBT-I would work to re-entrain the body's circadian rhythms. Alternatively, phase advancing the individual could reduce awakenings from a "sensitive period" in the circadian cycle, at which time an individual becomes depressed if they are awake. However, evidences for this hypothesis are relatively limited [40,41].

\section{Normalizing the HPA axis}

More and more studies have been devoted to looking for the mechanism underlying the co-morbidity and bidirectional relationship of insomnia and major depression. However, no conclusion has been drawn yet. One of the most widely accepted hypotheses regarding the pathophysiological basis of insomnia and major depression is the HPA axis dysfunction [42-44]. For example, the cortisol awakening response (CAR) of patients with severe depression disorder is significantly higher than that of normal controls [45], and the high level of CAR can predict the onset and recurrence of depressive episode $[46,47]$. Recently, we found that patients with insomnia also have hypercortisol arousal upon awakening [48]. In addition, it is also found that the cortisol and corticotrophin-releasing hormone levels of patients with primary insomnia were significantly higher than those of normal controls [42]. These results suggest that HPA axis dysfunction, including high cortisol arousal response, may be a common pathway of cause-and-effect relationship between insomnia disorder and major depression. In this regard, the mechanisms that CBT-I improves depressive symptoms seems to be via normalizing HPA axis activity in adolescents with both problems. However, further studies, which directly examine this assumption, are definitely needed.

\section{Regularizing neural processing}

Convergent evidences from neuroimaging research with healthy individuals shows that circuits involved in emotion regulation and circuits involved in sleep regulation interact in bidirectional ways at the neural systems level [49]. Depression and insomnia share neurobiological correlates such as sleep EEG profiles with regards to sleep continuity disturbances and slow wave sleep deficits [50,51]. By increasing slow wave sleep, the therapeutic effect of the behavioral components of CBT-I (sleep restriction and stimulus control) appears to result in an increase in homeostatic sleep drive [50]. At the behavioral level, one of the components of behavioral interventions, sleep restriction, may lead to increased time awake during the day to participate in planned pleasurable activities and improvement of depression through behavioral activation [51]. Therefore, CBT-I may regularize the central neural processing to improve sleep quality and decrease depressive symptoms.

\section{Reducing inflammation}

Another possible mechanism is related to inflammatory bio- markers. Sleep disturbance is associated with increased levels of inflammatory markers such as interleukin-1 beta and interleukin-6 in individuals with depression [52]. Treatment of insomnia using behavioral interventions leads to reductions in inflammatory markers [53].

\section{USING CBT-I TO TREAT DEPRESSION OR PREVENT DEPRESSION EPISODE IN ADOLESCENTS}

According to the comorbid and bidirectional relationship in depressive adolescents who experiences insomnia symptoms, and the possible mechanism of CBT-I treating insomnia to decrease depressive symptoms, we recommend that using CBT-I to treat the depressive adolescents who experience insomnia for the insufficient treatment on depression right now. This recommendation is in accordance with DSM-5 guidelines [54] in which treating insomnia is considered as a target for intervention itself.

Additional research should also attempt to elucidate the mechanism of action by which treating insomnia disorder leads to improvement in depression outcomes. This could include measuring inflammatory bio-markers, actigraphy, and measures of neurophysiology such as slow wave activity during sleep. This translational research could help further advance our understanding of the diseases themselves and focus on personalized approaches to treatment.

\section{CONCLUSION}

In summary, the current state of evidences suggest that in those with both insomnia disorder and, major depressive disorder, in addition to addressing depression, the treatment of insomnia should be an essential component in the overall treatment plan in order to maximize the likelihood of optimal clinical outcomes.

\section{Conflicts of Interest}

The authors have no potential conflicts of interest to disclose.

\section{Author Contributions}

Conceptualization: Weidong Song. Data collection: Xiaohui Hu. Formal analysis: Weidong Song. Funding acquisition: Beifang Fan. Investigation: Caihong Gao. Methodology: Weidong Song. Project administration: Caihong Gao. Resources: Mengshi Qiu. Software: Weidong Song. Supervision: Jihui Zhang. Validation: Jihui Zhang. Visualization: Jihui Zhang. Writing-original draft: Weidong Song. Writing_review \& editing: Jihui Zhang.

\section{ORCID iDs}

Jihui Zhang (1)

https://orcid.org/0000-0002-4976-3039

Weidong Song (1)

https://orcid.org/0000-0003-3798-3808 


\section{REFERENCES}

1. Zhang J, Li AM, Kong AP, Lai KY, Tang NL, Wing YK. A community-based study of insomnia in Hong Kong Chinese children: prevalence, risk factors and familial aggregation. Sleep Med 2009;10:1040-1046.

2. Patten CA, Choi WS, Gillin JC, Pierce JP. Depressive symptoms and cigarette smoking predict development and persistence of sleep problems in US adolescents. Pediatrics 2000;106:E23.

3. Morin CM, Benca R. Chronic insomnia. Lancet 2012;379:1129-1141.

4. Zhang B, Wing YK. Sex differences in insomnia: a meta-analysis. Sleep 2006; 29:85-93.

5. Fricke-Oerkermann L, Plück J, Schredl M, Heinz K, Mitschke A, Wiater A, et al. Prevalence and course of sleep problems in childhood. Sleep 2007;30: 1371-1377.

6. Nevéus T, Cnattingius S, Olsson U, Hetta J. Sleep habits and sleep problems among a community sample of schoolchildren. Acta Paediatr 2001;90:14501455.

7. Mong JA, Baker FC, Mahoney MM, Paul KN, Schwartz MD, Semba K, et al. Sleep, rhythms, and the endocrine brain: influence of sex and gonadal hormones. J Neurosci 2011;31:16107-16116.

8. Knutson KL. The association between pubertal status and sleep duration and quality among a nationally representative sample of U. S. adolescents. Am J Hum Biol 2005; 17:418-424.

9. Johnson EO, Roth T, Schultz L, Breslau N. Epidemiology of DSM-IV insomnia in adolescence: lifetime prevalence, chronicity, and an emergent gender difference. Pediatrics 2006;117:e247-e256.

10. Calhoun SL, Fernandez-Mendoza J, Vgontzas AN, Liao D, Bixler EO. Prevalence of insomnia symptoms in a general population sample of young children and preadolescents: gender effects. Sleep Med 2014;15:91-95.

11. Hagenauer MH, Perryman JI, Lee TM, Carskadon MA. Adolescent changes in the homeostatic and circadian regulation of sleep. Dev Neurosci 2009;31: 276-284.

12. Zhang J, Chan NY, Lam SP, Li SX, Liu Y, Chan JW, et al. Emergence of sex differences in insomnia symptoms in adolescents: a large-scale school-based study. Sleep 2016;39:1563-1570.

13. Schwarz SW. Adolescent mental health in the United States. New York: National Center for Children Poverty; 2009.

14. Born L, Shea A, Steiner M. The roots of depression in adolescent girls: is menarche the key? Curr Psychiatry Rep 2002;4:449-460.

15. Liu X, Buysse DJ, Gentzler AL, Kiss E, Mayer L, Kapornai K, et al. Insomnia and hypersomnia associated with depressive phenomenology and comorbidity in childhood depression. Sleep 2007;30:83-90.

16. Nierenberg AA, Husain MM, Trivedi MH, Fava M, Warden D, Wisniewski SR, et al. Residual symptoms after remission of major depressive disorder with citalopram and risk of relapse: a STAR*D report. Psychol Med 2010;40:41-50.

17. Sivertsen B, Harvey AG, Lundervold AJ, Hysing M. Sleep problems and depression in adolescence: results from a large population-based study of Norwegian adolescents aged 16-18 years. Eur Child Adolesc Psychiatry 2014;23: 681-689.

18. Roane BM, Taylor DJ. Adolescent insomnia as a risk factor for early adult depression and substance abuse. Sleep 2008;31:1351-1356.

19. Casement MD, Keenan KE, Hipwell AE, Guyer AE, Forbes EE. Neural reward processing mediates the relationship between insomnia symptoms and depression in adolescence. Sleep 2016;39:439-447.

20. Lovato N, Gradisar M. A meta-analysis and model of the relationship between sleep and depression in adolescents: recommendations for future research and clinical practice. Sleep Med Rev 2014;18:521-529.

21. de Zambotti M, Goldstone A, Colrain IM, Baker FC. Insomnia disorder in adolescence: diagnosis, impact, and treatment. Sleep Med Rev 2018;39:12-24.

22. American Psychological Association. Stress in America ${ }^{\mathrm{m}}$ : are teens adopting adults' stress habits? Available at: https://www.apa.org/news/press/releases/ stress/2013/stress-report.pdf. Accessed April 14, 2018

23. Liu S, Wing YK, Hao Y, Li W, Zhang J, Zhang B. The associations of long-time mobile phone use with sleep disturbances and mental distress in technical college students: a prospective cohort study. Sleep 2019;42:zsy213.

24. Hale L, Guan S. Screen time and sleep among school-aged children and adolescents: a systematic literature review. Sleep Med Rev 2015;21:50-58.

25. Johnson EO, Breslau N, Roehrs T, Roth, T. Insomnia in adolescence: epidemiology and associated problems. Sleep 1999;22(S1):s22.
26. Johnson EO, Roth T, Breslau N. The association of insomnia with anxiety disorders and depression: exploration of the direction of risk. J Psychiatr Res 2006; $40: 700-708$

27. Luo C, Zhang J, Pan J. One-year course and effects of insomnia in rural Chinese adolescents. Sleep 2013;36:377-384.

28. Emslie GJ, Armitage R, Weinberg WA, Rush AJ, Mayes TL, Hoffmann RF. Sleep polysomnography as a predictor of recurrence in children and adolescents with major depressive disorder. Int J Neuropsychopharmacol 2001;4: 159-168.

29. Kennard B, Silva S, Vitiello B, Curry J, Kratochvil C, Simons A, et al. Remission and residual symptoms after short-term treatment in the Treatment of Adolescents with Depression Study (TADS). J Am Acad Child Adolesc Psychiatry 2006;45:1404-1411.

30. March J, Silva S, Petrycki S, Curry J, Wells K, Fairbank J, et al. Fluoxetine, cognitive-behavioral therapy, and their combination for adolescents with depression: Treatment for Adolescents With Depression Study (TADS) randomized controlled trial. JAMA 2004;292:807-820.

31. Jaycox LH, Asarnow JR, Sherbourne CD, Rea MM, LaBorde AP, Wells KB. Adolescent primary care patients' preferences for depression treatment. Adm Policy Ment Health 2006;33:198-207.

32. Sivertsen B, Omvik S, Pallesen S, Bjorvatn B, Havik OE, Kvale G, et al. Cognitive behavioral therapy vs zopiclone for treatment of chronic primary insomnia in older adults: a randomized controlled trial. JAMA 2006;295:28512858.

33. Cunningham JEA, Shapiro CM. Cognitive Behavioural Therapy for Insomnia (CBT-I) to treat depression: a systematic review. J Psychosom Res 2018; 106:1-12.

34. Perach R, Allen CK, Kapantai I, Madrid-Valero JJ, Miles E, Charlton RA, et al. The psychological wellbeing outcomes of nonpharmacological interventions for older persons with insomnia symptoms: a systematic review and meta-analysis. Sleep Med Rev 2019;43:1-13.

35. Clarke G, McGlinchey EL, Hein K, Gullion CM, Dickerson JF, Leo MC, et al. Cognitive-behavioral treatment of insomnia and depression in adolescents: a pilot randomized trial. Behav Res Ther 2015;69:111-118.

36. Conroy DA, Czopp AM, Dore-Stites DM, Dopp RR, Armitage R, Hoban TF, et al. Modified cognitive behavioral therapy for insomnia in depressed adolescents: a pilot study. Behav Sleep Med 2019;17:99-111.

37. Palermo TM, Beals-Erickson S, Bromberg M, Law E, Chen M. A single arm pilot trial of brief cognitive behavioral therapy for insomnia in adolescents with physical and psychiatric comorbidities. J Clin Sleep Med 2017;13:401410.

38. de Bruin EJ, Bögels SM, Oort FJ, Meijer AM. Improvements of adolescent psychopathology after insomnia treatment: results from a randomized controlled trial over 1 year. J Child Psychol Psychiatry 2018;59:509-522.

39. McClung CA. How might circadian rhythms control mood? Let me count the ways...Biol Psychiatry 2013;74:242-249.

40. Wu JC, Bunney WE. The biological basis of an antidepressant response to sleep deprivation and relapse: review and hypothesis. Am J Psychiatry 1990; 147:14-21.

41. Germain A, Kupfer DJ. Circadian rhythm disturbances in depression. Hum Psychopharmacol 2008;23:571-585.

42. Xia L, Chen GH, Li ZH, Jiang S, Shen J. Alterations in hypothalamus-pituitary-adrenal/thyroid axes and gonadotropin-releasing hormone in the patients with primary insomnia: a clinical research. PLoS One 2013;8:e71065.

43. Riemann D, Spiegelhalder K, Feige B, Voderholzer U, Berger M, Perlis M, et al. The hyperarousal model of insomnia: a review of the concept and its evidence. Sleep Med Rev 2010;14:19-31.

44. Bonnet MH, Arand DL. Hyperarousal and insomnia: state of the science. Sleep Med Rev 2010;14:9-15.

45. Young E, Korszun A. Sex, trauma, stress hormones and depression. Mol Psychiatry 2010;15:23-28.

46. Vrshek-Schallhorn S, Doane LD, Mineka S, Zinbarg RE, Craske MG, Adam EK. The cortisol awakening response predicts major depression: predictive stability over a 4-year follow-up and effect of depression history. Psychol Med 2013;43:483-493

47. Adam EK, Doane LD, Zinbarg RE, Mineka S, Craske MG, Griffith JW. Prospective prediction of major depressive disorder from cortisol awakening responses in adolescence. Psychoneuroendocrinology 2010;35:921-931.

48. Zhang J, Lam SP, Li SX, Ma RC, Kong AP, Chan MH, et al. A community- 
based study on the association between insomnia and hypothalamic-pituitary-adrenal axis: sex and pubertal influences. J Clin Endocrinol Metab 2014; 99:2277-2287.

49. Saper CB, Cano G, Scammell TE. Homeostatic, circadian, and emotional regulation of sleep. J Comp Neurol 2005;493:92-98.

50. Krystal AD, Edinger JD. Sleep EEG predictors and correlates of the response to cognitive behavioral therapy for insomnia. Sleep 2010;33:669-677.

51. Staner L. Comorbidity of insomnia and depression. Sleep Med Rev 2010;14: 35-46.

52. Chan J, Li A, So K, Chen J, Yuen L, Chung K, et al. 1087: QIGONG Exercise improved quality of sleep and reduced interleukin- 1 beta and interleukin- 6 among presons with depressive symptoms and sleep disturbances: a randomized controlled trial. Sleep 2017;40(suppl_1):A405.

53. Irwin MR, Olmstead R, Carrillo C, Sadeghi N, Breen EC, Witarama T, et al. Cognitive behavioral therapy vs. Tai Chi for late life insomnia and inflammatory risk: a randomized controlled comparative efficacy trial. Sleep 2014 37:1543-1552.

54. American Psychiatric Association. Diagnostic and statistical manual of mental disorders $\left(\mathrm{DSM}-5^{\circledR}\right)$. Arlington (VA): American Psychiatric Pub; 2013. 